\title{
Ractopamine supplementation improves leanness and carcass yield, minimally affecting pork quality in immunocastrated pigs
}

\author{
Osmar Antonio Dalla Costaㄹ ${ }^{1}$ Vivian Feddern ${ }^{1}$, Natalia Bortoleto Athayde ${ }^{2}$, Naiana Einhardt Manzke ${ }^{3}$, Roberto de Oliveira Roça ${ }^{4}$, Letícia \\ dos Santos Lopes ${ }^{1}$, Gustavo Julio Mello Monteiro de Lima ${ }^{1 *}$
}

${ }^{1}$ Embrapa Swine and Poultry, Rod. BR 153, km 110 89715-899 - Concórdia, SC - Brazil.

2Embrapa Acre, Rod. BR 364, km 14 - 69900-970 - Rio

Branco, AC - Brazil.

${ }^{3}$ Kerry do Brasil Ltda, Av. Mercedes Benz, 460 - 13054-750

- Campinas, SP - Brazil.

${ }^{4}$ São Paulo State University/FCA - Economy, Sociology and Technology Dept., C.P. 237 - 18603-970 - Botucatu, SP Brazil.

*Corresponding author <gustavo.lima@embrapa.br>

Edited by: Gerson Barreto Mourão

Received September 05, 2016

Accepted February 21, 2017
ABSTRACT: This study was carried out to determine the combining effects of feeding up to 15 $\mathrm{mg} \mathrm{kg}^{-1}$ ractopamine (RAC) to physically castrated (PC) pigs or those injected with an anti-GnRF vaccine (IC), on growth performance during suckling, weaning, growing and finishing phases. Out of a total of 1,160 male piglets, $50 \%$ were surgically castrated at age 7 , while $50 \%$ underwent immunization against GnRF on the $103^{\text {rd }}$ and $136^{\text {th }}$ day of life. A completely randomized design, with two treatments (PC and IC), was used from the $1^{\text {st }}$ until the $60^{\text {th }}$ day of life. Afterwards, 792 animals, PC and IC, selected according to overall average weight $(23.3 \pm 0.69 \mathrm{~kg})$ were allotted based on a completely randomized block design with a $2 \times 4$ factorial arrangement of treatments (castration categories $\times$ RAC levels) with 9 replications (11 animals) per main combination effect. IC growing pigs improved feed conversion due to lower average daily feed intake and higher average daily gain (ADG). During the finishing phase, both RAC and immunocastration increased body weight, but the effects were independent. RAC, on its own improved ADG and feed conversion. However, pigs fed $10 \mathrm{mg} \mathrm{kg}^{-1} \mathrm{RAC}$ had higher blood lactate compared to those fed lower levels, suggesting that these animals were more susceptible to stress. Muscle depth was influenced only by RAC, whereas fat thickness was lower for IC. Lean meat increased and fat decreased in the shoulder and belly of IC; RAC had a positive effect on belly lean meat only. Either immunocastration or RAC inclusion (at least $5 \mathrm{mg} \mathrm{kg}^{-1}$ ) promoted muscle gain and fat reduction in pig carcass, adding value to pork.

Keywords: $\beta$-agonists, cutting yield, lean meat, stress parameters

\section{Introduction}

The meat market demands high nutritional value products with low fat content, suitable tenderness and attractiveness to the most selective consumers. The challenge also includes meat production within the ethical principles of animal welfare at an affordable cost and a profitable return. An immunocastrated pig (IC) is a boar immunized against gonadotrophin-releasing factor $(\mathrm{GnRF})$. This practice, together with the addition of ractopamine, is one technological innovation available to achieve these goals.

Ractopamine hydrochloride (RAC) is used as a repartitioning agent in pig diets 28 days before slaughter to increase muscle protein synthesis (Ulrey et al., 2013), improve animal performance (Puls et al., 2014) and reduce carcass fat (Moore et al., 2009) without altering meat quality (Elsbernd et al., 2015). Swine nutritionists elected RAC in a survey as the $13^{\text {th }}$ most important technological innovation out of 20 that comes to mind when considering the top nutritional discoveries of the past century (Cromwell, 2009).

Both surgical castration and immunocastration are necessary practices for the control of boar taint in pork, which is caused mainly by androstenone pheromone and skatole. This boar taste is highly undesirable to consumers (Meier-Dinkel et al., 2013). Surgical castration has recently been questioned by both governmental authorities and animal welfare groups, since castration is prac- ticed without anesthesia, which is potentially painful for piglets (Aaslyng et al., 2016). Immunization against GnRF inhibits testicular function, improves growth and carcass quality, and, additionally, reduces stress and handling compared to surgical castration (Rikard-Bell et al., 2009). Despite the positive effects of immunocastration, the cost of this technology merits attention which includes investment and handling with two single vaccine applications.

Given this scenario, RAC becomes more economically viable as it amplifies immunocastration benefits by allowing pigs to perform as intact males until the second vaccination (Asmus et al., 2014), altering feed intake and tissue deposition thereafter (Dunshea et al., 2013). Moreover, both practices are additive in terms of improving growth performance and carcass characteristics (Lowe et al., 2014).

Thus, the aim of this study was to evaluate the effects of increasing levels of RAC supplementation in the diet on PC and IC pigs in terms of animal growth performance, carcass traits and meat quality.

\section{Materials and Methods}

The experimental protocol was developed in accordance with the ethical principles of animal experimentation as determined by the Ethical Committee on Animal Experimentation of the São Paulo State University (UNESP), Botucatu, SP, Brazil (protocol no.: 99/2010-CEUA). 


\section{Animals, housing, and handling}

The experiment was conducted in commercial facilities, from the same integrator, located in the Midwest Region of the state of Santa Catarina, Brazil

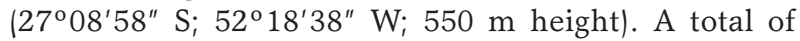
1,160 male piglets (Camborough $25 \times$ AGPIC 337) were produced in 17 farms and selected according to weight $(23.3 \pm 0.69 \mathrm{~kg})$ and birth within the same week. These genetic lines were chosen because they are directed to meat production and low fat deposition.

Surgical castration was performed on half of the littermates on the $7^{\text {th }}$ day of life whereas the remaining animals were immunocastrated through two vaccinations on the $103^{\text {rd }}$ and $136^{\text {th }}$ days of age using a safety vaccination gun at a dose of $2 \mathrm{~mL}$ of commercial vaccine $10.4 \mathrm{mg}$ of $2-10 \mathrm{GnRF}$ conjugated to Diphtheria Toxoid) behind and below the ear base. The chronology of the experiment is illustrated in Figure 1.

At weaning, on day 27, mortalities and runt piglets accounted for a total of 56 piglets (PC $=43$ and IC $=13$ ). All animals from the 17 farms were weighed at weaning, and the remaining 1,104 animals were transferred to a single nursery and allotted by castration category to 40 pens of 27 or 28 pigs. The end of the initial phase was at $60 \mathrm{~d}$ of age, when all pigs were weighed again. Based on castration category and blocking for similar weight, 792 pigs were transferred to a growing-finishing facility divided into 72 pens of 11 animals each, according to a randomized complete block design (RCBD), in a $2 \times 4$ factorial arrangement (two castration categories $\times$ four levels of RAC inclusion) with nine replications per combination of main factors.

Mash diets had the same ingredient composition throughout the experiment, except for the RAC inclusion levels which were only fed for 28 days prior to slaughter (Table 1).

\section{Performance evaluation, pre-slaughter, and slaughter handling}

Pig performance was evaluated during the initial phase (0 to 27 days), the nursery (28 to 60 days), the growing and at part of the finishing phase (61 to 136 days), and the end of finishing phase (137 to 164 days), when the animals were fed RAC diets.

Feed was withheld for $4 \mathrm{~h}$ before pigs ( $\mathrm{n}=792$ ) were weighed and transported to a packing plant in Chapecó, SC, Brazil (2705'47" S; 52 ${ }^{\circ} 37^{\prime} 06^{\prime \prime}$ W; $674 \mathrm{~m}$ height). The animals were held in pens for $6 \mathrm{~h}$ before being electrically stunned, exsanguinated from major blood vessels near the heart, and processed according to industry-accepted procedures.

Table 1 - Ingredient and calculated composition of experimental diets provided 28 days prior to slaughter.

\begin{tabular}{|c|c|c|c|c|}
\hline \multirow{2}{*}{ Item } & \multicolumn{4}{|c|}{ Ractopamine $\left(\mathrm{mg} \mathrm{kg}^{-1}\right)$} \\
\hline & 0 & 5 & 10 & 15 \\
\hline \multicolumn{5}{|l|}{ Ingredients $\left(\mathrm{g} \mathrm{kg}^{-1}\right)$} \\
\hline Corn & 716.3 & 716.1 & 715.8 & 715.6 \\
\hline Soybean meal & 236.0 & 236.0 & 236.0 & 236.0 \\
\hline Soybean oil & 16.0 & 16.0 & 16.0 & 16.0 \\
\hline Dicalcium phosphate & 5.5 & 5.5 & 5.5 & 5.5 \\
\hline Limestone & 7.7 & 7.7 & 7.7 & 7.7 \\
\hline L-Lys HCl & 1.6 & 1.6 & 1.6 & 1.6 \\
\hline DL-Met & 0.7 & 0.7 & 0.7 & 0.7 \\
\hline Salt & 3.6 & 3.6 & 3.6 & 3.6 \\
\hline Vitamin premix and trace mineral ${ }^{1}$ & 10.0 & 10.0 & 10.0 & 10.0 \\
\hline Mycotoxin adsorbent ${ }^{2}$ & 2.5 & 2.5 & 2.5 & 2.5 \\
\hline Ractopamine hydrocloride & 0.0 & 0.25 & 0.50 & 0.75 \\
\hline \multicolumn{5}{|l|}{ Calculated composition ${ }^{3}$} \\
\hline $\mathrm{CP}\left(\mathrm{g} \mathrm{kg}^{-1}\right)$ & 167.9 & 167.8 & 167.8 & 167.8 \\
\hline ME (kcal kg-1) & 3,277 & 3,276 & 3,275 & 3,275 \\
\hline Total Lys ( $\mathrm{g} \mathrm{kg}^{-1}$ ) & 9.9 & 9.9 & 9.9 & 9.9 \\
\hline SID Lys (g kg-1) & 8.5 & 8.5 & 8.5 & 8.5 \\
\hline Available $\mathrm{P}\left(\mathrm{g} \mathrm{kg}^{-1}\right)$ & 1.8 & 1.8 & 1.8 & 1.8 \\
\hline $\mathrm{Ca}\left(\mathrm{g} \mathrm{kg}^{-1}\right)$ & 5.1 & 5.1 & 5.1 & 5.1 \\
\hline \multicolumn{5}{|c|}{ 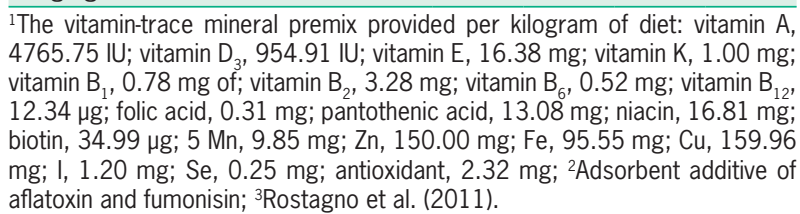 } \\
\hline
\end{tabular}

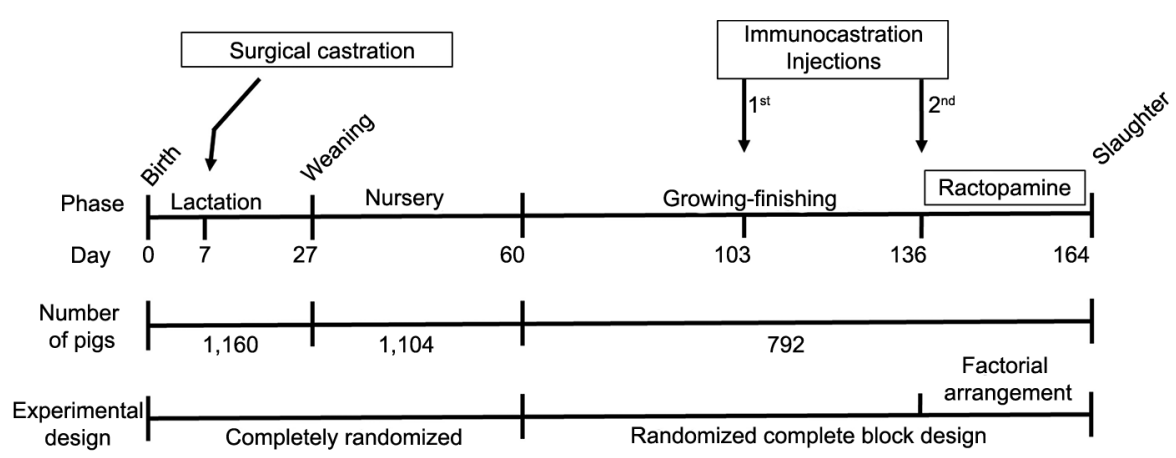

Figure 1 - Scheme showing when both castrations were performed, the number of pigs in each phase and the experimental design applied. 


\section{Stress parameters}

Two pigs from each pen were chosen based on their live weight, closest to pen average final weight, and had blood samples collected on the $164^{\text {th }}$ day of experiment, in a horizontal position as bleeding began during slaughter. Samples were collected in conical tubes containing 2,500 UI of fluoride anticoagulant. Later, they were centrifuged and frozen at $-30{ }^{\circ} \mathrm{C}$ for subsequent biochemical analysis of stress parameters (cortisol and lactate). Cortisol was determined by radioimmunoassay and dosed in a gamma counter. Lactate determination was done under $546 \mathrm{~nm}$ and $340 \mathrm{~nm}$ wavelengths using a spectrophotometer.

\section{Carcass trait evaluation}

Hot carcasses from all 792 pigs were weighed and optically probed (Hennessy Grading System - GP4 / BP4) across a $6 \mathrm{~cm}$ insertion on the median dorsal line of the carcass, between the last and the penultimate rib in order to measure backfat thickness $(\mathrm{BF})$ and Longissimus dorsi (LD) depth. These variables and hot carcass weight (HCW) were used to estimate the lean meat percentage (LMP) through the equation LMP $=58.408-(0.5886 *$ $\mathrm{BF})+(0.1739 * \mathrm{LD}$ depth $)-(0.0189 * \mathrm{HCW})$. For each cut, carcass yield was expressed as $\mathrm{g} 100 \mathrm{~g}^{-1}$ of the hot carcass weight, dividing the corresponding cut by the live weight at slaughter.

Ham and loin quality traits, as well as marbling evaluation, were measured in samples collected from the same two animals/pen, used for blood collection. Semimembranosus muscle (SM) pH was measured between the tail head and the tuber ischium, by inserting the $\mathrm{pH}$ probe $3.5 \mathrm{~cm}$ into the SM. Loin $\mathrm{pH}$ was measured at $45 \mathrm{~min}(\mathrm{pHi})$ and $24 \mathrm{~h}$ postmortem $(\mathrm{pHu})$ with a temperature-compensating electrode attached to a $\mathrm{pH}$ meter.

After a $24 \mathrm{~h}$ chilling period, instrumental color was determined on a center-cut SM slice after a $30 \mathrm{~min}$ bloom period. Instrumental color $\left(\mathrm{L}^{*}, \mathrm{a}^{*}\right.$, and $\mathrm{b}^{*}$ values) was measured with a Konica-Minolta colorimeter using DL65 illuminant and a $0^{\circ}$ viewing angle. In addition, marbling was visually evaluated in the LD using the NPPC (1999) marbling standards ( $1=1 \%$ intramuscular fat to $10=10 \%$ intramuscular fat).

\section{Organs and testis weight and yield}

All animals from each pen had their organs evaluated. Immediately after slaughter, gastrointestinal tract, liver, heart, kidneys and testicles (without epididymis) were removed, identified, cleaned (removing surface fat), and weighed. Organs and testicle yield were calculated dividing the HCW by each tissue weight, expressed as $\mathrm{g}_{100 \mathrm{~g}^{-1} \text {. }}$.

\section{Carcass cutting yield}

One animal from each pen was selected based on closest weight to the pen average in order to have cuts evaluated. Carcasses were dissected after being subject- ed to temperatures ranging between 1 and $4{ }^{\circ} \mathrm{C}$ for $24 \mathrm{~h}$. Carcass left side was divided into five cuts (ham, loin, belly + ribs, shoulder, and Boston butt) in order to determine each cut yield (weight of the component, divided by hot carcass weight). Cuts were weighed, deboned, and manually dissected to find the weights of lean tissue, subcutaneous fat, and skin. These procedures were performed by the same person who was blinded to the treatment assignment. Lean meat, fat, skin, and bone yield were calculated by section weight, divided by corresponding cut, expressed as $\mathrm{g} 100 \mathrm{~g}^{-1}$.

\section{Pork meat quality}

Duplicate samples of LD were collected between the $6^{\text {th }}$ and $7^{\text {th }}$ ribs, $24 \mathrm{~h}$ after slaughter, and weighed before being placed into EX-DripLoss tubes in order to measure drip loss according to Athayde et al. (2012). Tubes were stored at $4{ }^{\circ} \mathrm{C}$ for $48 \mathrm{~h}$ before muscles were removed from the tubes and reweighed. The difference between the initial and $48 \mathrm{~h}$ muscle sample weights was divided by the initial sample weight (Honikel, 1998). Furthermore, LD duplicate samples were collected 24 $\mathrm{h}$ after slaughter, placed in freezer temperatures until they were ground in a microprocessor, packaged in petri dishes, and the intramuscular fat (IMF) content was determined by ether extraction AOAC method 960.39 number 39.1.05 (AOAC, 2007). In addition, LD samples were collected and cut into four $2.5 \mathrm{~cm}$ thick chops, which were packaged, frozen, and stored at $-20{ }^{\circ} \mathrm{C}$. These frozen LD chops were allowed to thaw for $24 \mathrm{~h}$ at $5{ }^{\circ} \mathrm{C}$ before duplicate $100 \mathrm{~g}$ samples were vacuumpackaged and cooked in an $80{ }^{\circ} \mathrm{C}$ water bath for $1 \mathrm{~h}$. After cooking, samples were placed on absorbent paper to equilibrate to room temperature $\left(20\right.$ to $\left.23^{\circ} \mathrm{C}\right)$, and subsequently reweighed to calculate cooking loss percentage. Then, five $1.0 \mathrm{~cm} \times 1.0 \mathrm{~cm} \times$ chop thickness cubes were cut from each sample, and each cube was sheared perpendicular to the muscle fiber orientation with a Warner-Bratzler shear force (WBSF) device attached to a texture analyzer equipped with a $25 \mathrm{~kg}$ load cell and crosshead speed of $200 \mathrm{~mm} / \mathrm{min}$ (AMSA, 1995).

\section{Statistical analysis}

A completely randomized design with two treatments (PC and IC) was considered from birth until the end of the nursery phase (at day 60). Means were compared by $F$ test after running the PROC MIXED procedure in the SAS 9.1 (2002-2003) program.

From the beginning of the growing phase until slaughter (from day 61 to day 164), a factorial design (two castration categories $\times$ four levels of RAC inclusion) was adopted being inherent in the RCBD. The criterion of block formation was animal weight at 60 days of age and the ANOVA model was: $y_{j i k}=\mu+b_{j}+c_{i}+r_{k}+e t_{i k}+\sum_{j i k}$ i being $j=1,2, \ldots, 9$ weight ranges; $i=1,2$ castration categories (castrates and immunocastrates); $k=1,2,3,4$ RAC inclusion levels; 
where: $y_{j i k}$ is the observation of the response belonging to the $j$ weight category, $i$ castration category e $k$ RAC level; $b_{j}$ the weight category (light, medium and heavy) effect (blocking factor); $c_{i}$ the effect of castration category; $r_{k}$ the effect of RAC level; $e t_{i k}$ the interaction (castration vs. $\mathrm{RAC}$ ) effect; $e_{j i k}$ the residual error following the normal distribution with mean equal to zero and constant variance $\int^{2}$.

To determine if two sets of data were significantly different from each other, means were compared using Student's t-test. Differences were considered significant at the level of $p<0.05$.

\section{Results}

Table 2 shows the performance parameters during the suckling and weaning periods in castrated pigs. There were no interactions $(p>0.05)$ between RAC and castration category during the finishing period.

Immunocastration was efficient in reducing $(p=$ $0.016)$ average daily feed intake (ADFI), and increasing $(p<0.001)$ ADG during the growing period, leading to a better feed conversion $(p<0.001)$ compared to PC pigs.

During the finishing period, IC pigs had higher body weight (BW) and also final BW $(p<0.001)$ but there were no differences in ADFI, ADG and feed conversion, compared to the PC category. In contrast, RAC inclusion in the diet during this phase resulted in higher ADG $(p<0.001)$ and final BW $(p=0.011)$, regardless of the given level. RAC improved feed conversion $(p<0.001)$ at all levels compared to pigs fed a diet without RAC. Pigs fed $15 \mathrm{mg} \mathrm{kg}^{-1} \mathrm{RAC}$ had a better feed conversion response than pigs fed $5 \mathrm{mg} \mathrm{kg}^{-1}$; however, they were similar to those fed $10 \mathrm{mg} \mathrm{kg}^{-1}$.

Castration categories had no influence on stress parameters (Table 2), and RAC levels did not affect $(p>0.10)$ the cortisol concentration in the pigs. However, pigs fed $10 \mathrm{mg} \mathrm{kg}$ of RAC showed higher $(p=$ $0.012)$ lactate concentration compared to pigs fed $5 \mathrm{mg}$ $\mathrm{kg}^{-1}$ of RAC and the control group, with no differences between pigs receiving $15 \mathrm{mg} \mathrm{kg}^{-1}$ of RAC.

There was no interaction $(p>0.05)$ between castration category and RAC level for carcass traits; therefore, only the main effects are presented (Table 3). IC pigs had heavier HCW ( $p=0.004)$, higher lean percentage $(p=0.007)$ and lower backfat thickness $(p<0.001)$, than PC pigs, although PC pigs had higher carcass yield $(p<0.001)$. Pigs fed RAC levels showed higher HCW $(p<0.001)$, loin depth $(p=0.003)$, and lean percentage $(p=0.002)$, regardless of the level used in the diet, than pigs fed diets without RAC. Carcass yield increased with the RAC dosage $(p<0.001)$, whereas pigs fed $15 \mathrm{mg} \mathrm{kg}^{-1}$ of RAC had higher carcass yield than pigs receiving 0 and $5 \mathrm{mg} \mathrm{kg}^{-1}$.

Table 2 - Effect of castration categories ${ }^{1}$ and levels of RAC ${ }^{2}$ in the diet on growth performance and stress parameters in pigs.

\begin{tabular}{|c|c|c|c|c|c|c|c|c|c|c|c|}
\hline \multirow{2}{*}{ Item } & \multicolumn{4}{|c|}{ Ractopamine $\left(\mathrm{mg} \mathrm{kg}^{-1}\right)$} & \multirow{2}{*}{ SEM } & \multicolumn{2}{|c|}{ Castration category } & \multirow{2}{*}{ SEM } & \multicolumn{3}{|r|}{$p$-value } \\
\hline & 0 & 5 & 10 & 15 & & PC & IC & & RAC & Castration & RAC $\times$ Castration interaction \\
\hline \multicolumn{12}{|l|}{ Suckling period ${ }^{3}$} \\
\hline Birthweight (kg) & - & - & - & - & - & 1.79 & 1.81 & 0.07 & - & 0.778 & - \\
\hline ADFI $\left(\mathrm{kg} \mathrm{d}^{-1}\right)$ & - & - & - & - & - & 0.037 & 0.033 & 0.100 & - & 0.590 & - \\
\hline Weaning weight at $\mathrm{d} 27\left(\mathrm{~kg} \mathrm{~d}^{-1}\right)$ & - & - & - & - & - & 7.89 & 7.82 & 0.35 & - & 0.865 & - \\
\hline \multicolumn{12}{|l|}{ Weaning to $60 \mathrm{~d}^{4}$} \\
\hline Final weight (kg) & - & - & - & - & - & 23.40 & 23.20 & 0.70 & - & 0.807 & - \\
\hline $\operatorname{ADFI}\left(\mathrm{kg} \mathrm{d}^{-1}\right)$ & - & - & - & - & - & 0.664 & 0.643 & 0.018 & - & 0.507 & - \\
\hline $\mathrm{ADG}\left(\mathrm{kg} \mathrm{d}^{-1}\right)$ & - & - & - & - & - & 0.471 & 0.465 & 0.013 & - & 0.775 & - \\
\hline Feed conversion & - & - & - & - & - & 1.413 & 1.383 & 0.035 & - & 0.515 & - \\
\hline \multicolumn{12}{|l|}{ Growing period (61 to $136 \mathrm{~d})^{5}$} \\
\hline Initial BW (kg) & - & - & - & - & - & 24.09 & 23.94 & 0.57 & - & 0.830 & - \\
\hline ADFI $\left(\mathrm{kg} \mathrm{d}^{-1}\right)$ & - & - & - & - & - & 1.900 & 1.857 & 0.027 & - & 0.016 & - \\
\hline $\operatorname{ADG}\left(\mathrm{kg} \mathrm{d}^{-1}\right)$ & - & - & - & - & - & 0.861 & 0.921 & 0.009 & - & $<0.001$ & - \\
\hline Feed conversion & - & - & - & - & - & 2.207 & 2.014 & 0.019 & - & $<0.001$ & - \\
\hline \multicolumn{12}{|l|}{ Finishing period (137 to $164 d)^{5}$} \\
\hline BW at 136 days (kg) & 92.06 & 92.11 & 91.57 & 91.14 & 1.96 & 89.50 & 93.93 & 1.23 & 0.557 & $<0.001$ & 0.934 \\
\hline ADFI $\left(\mathrm{kg} \mathrm{d}^{-1}\right)$ & 2.745 & 2.726 & 2.679 & 2.684 & 0.063 & 2.707 & 2.711 & 0.040 & 0.319 & 0.906 & 0.945 \\
\hline $\mathrm{ADG}\left(\mathrm{kg} \mathrm{d}^{-1}\right)$ & $0.864^{b}$ & $0.970^{a}$ & $0.991^{\mathrm{a}}$ & $1.017^{\mathrm{a}}$ & 0.020 & 0.966 & 0.954 & 0.017 & $<0.001$ & 0.484 & 0.732 \\
\hline Feed conversion & $3.199^{a}$ & $2.818^{b}$ & $2.704^{b c}$ & $2.640^{c}$ & 0.081 & 2.818 & 2.863 & 0.055 & $<0.001$ & 0.359 & 0.903 \\
\hline Final BW (kg) & $116.24^{b}$ & $119.25^{\mathrm{a}}$ & $119.3^{a}$ & $119.62^{\mathrm{a}}$ & 2.29 & 116.56 & 120.65 & 1.46 & 0.011 & $<0.001$ & 0.747 \\
\hline \multicolumn{12}{|l|}{ Stress parameters ${ }^{6}$} \\
\hline Cortisol ( $\left.\mu \mathrm{g} \mathrm{dL}^{-1}\right)$ & 6.98 & 7.01 & 7.02 & 6.81 & 0.30 & 7.05 & 6.87 & 0.19 & 0.893 & 0.543 & 0.982 \\
\hline Lactate (mmol L-1) & $10.86^{b c}$ & $10.14^{c}$ & $13.59^{a}$ & $12.80^{\mathrm{ab}}$ & 0.93 & 11.25 & 12.37 & 0.60 & 0.012 & 0.217 & 0.595 \\
\hline
\end{tabular}

${ }^{1} \mathrm{PC}=$ physically castrated (at age 7); IC $=$ immunocastrated on $103^{\text {rd }}$ and $136^{\text {th }}$ day of life; $A D F I=$ average daily feed intake; $A D G=$ average daily gain; $B W=$ body weight; ${ }^{2} \mathrm{RAC}=$ Ractopamine-HCl; ${ }^{3}$ Considering initial $\mathrm{n}=1,160$ piglets; ${ }^{4}$ Considering initial $\mathrm{n}=1,104$ piglets; ${ }^{5}$ Considering initial $\mathrm{n}=792$ pigs; ${ }^{6} \mathrm{Blood}$ samples were collected at preslaughter; a.cWithin a row, means without a common superscripts differ $(p<0.05)$ among RAC levels by Student's t-test. 
Results of ham and loin quality traits are also presented in Table 3. There was no difference $(p>0.05)$ in RAC levels or castration category on ham $\mathrm{pH}$ and initial temperature. Ham ultimate temperature was higher $(p=0.013)$ for PC pigs; however, it was not affected by RAC levels $(p>0.05)$. Pigs fed $5 \mathrm{mg} \mathrm{kg}^{-1}$ of RAC showed higher ham lightness $\left(\mathrm{L}^{*} ; p=0.030\right)$ compared to other treatments. Ham redness $\left(\mathrm{a}^{*}\right)$ color was lower $(p$ $=0.030$ ) in pigs receiving 5 and $15 \mathrm{mg} \mathrm{kg}^{-1}$ of RAC; however, pigs fed $10 \mathrm{mg} \mathrm{kg}^{-1}$ of RAC a* did not differ from control pigs. RAC had no influence ( $p=0.098)$ on ham yellowness $\left(b^{*}\right)$. Immunocastration showed no effect on ham color attributes.

Loin quality traits (Table 3), L* (lightness) values were not influenced by RAC or castration category $(p>$ $0.05)$. Pigs fed RAC had increased ( $p=0.001)$ a* (redness) regardless of the inclusion level; however, no effect in $\mathrm{b}^{*}$ (yellowness) values were observed $(p>0.05)$. IC pigs presented lower $\mathrm{a}^{*}(p=0.008)$ and $\mathrm{b}^{*}(p=0.028)$ compared to PC pigs. RAC level and castration category had no effects $(p>0.05)$ on loin drip loss and cooking loss. Shear force of loin was also not affected by castration category, however it did increase $(p=0.001)$ with RAC inclusion regardless of the supplemented level. The RAC level did not affect marbling score ( $p=0.276)$, however indicated a tendency to decrease IMF content $(p=0.077)$; IC pigs had their IMF decreased $(p<0.001)$ and their marbling score tended $(p=0.074)$ to decrease.

IC pigs had no alteration in organ yield $(p>0.05)$, with the exception of liver yield which increased $(p<0.001)$ in these pigs (Table 4$)$. Liver yield was also influenced by RAC level, as pigs fed $10 \mathrm{mg} \mathrm{kg}^{-1} \mathrm{RAC}$ showed lower $(p=0.010)$ liver weight compared to those pigs receiving 0 and $5 \mathrm{mg} \mathrm{kg}^{-1}$ of RAC.

For all studied variables, no interaction effect $(p>0.05)$ was observed between RAC and castration category. None of the castration categories and neither the RAC level had an effect $(p>0.05)$ on the following

Table 3 - Effect of castration categories ${ }^{1}$ and levels of $\mathrm{RAC}^{2}$ in the diet on pig carcass traits and quality parameters of meat.

\begin{tabular}{|c|c|c|c|c|c|c|c|c|c|c|c|}
\hline \multirow{2}{*}{ Item } & \multicolumn{4}{|c|}{ Ractopamine $\left(\mathrm{mg} \mathrm{kg}^{-1}\right)$} & \multirow{2}{*}{ - SEM } & \multicolumn{2}{|c|}{ Castration category } & \multirow{2}{*}{ SEM } & \multicolumn{3}{|r|}{$p$-value } \\
\hline & 0 & 5 & 10 & 15 & & PC & IC & & RAC & Castration & RAC $\times$ Castration Interaction \\
\hline \multicolumn{12}{|l|}{ Slaughter traits ${ }^{3}$} \\
\hline HCW (kg) & $84.80^{\mathrm{b}}$ & $87.70^{a}$ & $88.24^{a}$ & $88.70^{\mathrm{a}}$ & 1.74 & 86.56 & 88.16 & 1.13 & $<0.001$ & 0.004 & 0.923 \\
\hline Carcass yield $\left(\mathrm{g} \mathrm{kg}^{-1}\right)^{4}$ & $729.6^{c}$ & $735.5^{\mathrm{bc}}$ & $739.3^{\mathrm{ab}}$ & $743.3^{a}$ & 0.34 & 743.7 & 730.2 & 0.19 & $<0.001$ & $<0.001$ & 0.473 \\
\hline Backfat (mm) & 15.73 & 15.31 & 15.17 & 15.27 & 0.46 & 15.98 & 14.77 & 0.31 & 0.349 & $<0.001$ & 0.161 \\
\hline Loin depth (mm) & $60.68^{b}$ & $63.37^{a}$ & $63.78^{a}$ & $63.38^{a}$ & 0.74 & 63.24 & 62.36 & 0.53 & 0.003 & 0.167 & 0.814 \\
\hline Leanness $\left(\mathrm{g} \mathrm{kg}^{-1}\right)^{5}$ & $576.3^{b}$ & $584.0^{\mathrm{a}}$ & $585.6^{a}$ & $584.2^{a}$ & 0.23 & 58.00 & 58.51 & 0.18 & 0.002 & 0.007 & 0.386 \\
\hline \multicolumn{12}{|l|}{ Ham quality traits ${ }^{6}$} \\
\hline \multicolumn{12}{|l|}{$\mathrm{pH}^{7}$} \\
\hline Initial pH & 6.40 & 6.46 & 6.41 & 6.41 & 0.03 & 6.42 & 6.42 & 0.02 & 0.542 & 0.918 & 0.803 \\
\hline Ultimate $\mathrm{pH}$ & 5.24 & 5.24 & 5.26 & 5.26 & 0.01 & 5.26 & 5.24 & 0.01 & 0.433 & 0.166 & 0.511 \\
\hline \multicolumn{12}{|l|}{ Temperature $\left({ }^{\circ} \mathrm{C}\right)^{7}$} \\
\hline Initial temperature & 32.16 & 32.26 & 32.49 & 32.38 & 0.18 & 32.29 & 32.24 & 0.13 & 0.399 & 0.890 & 0.158 \\
\hline Ultimate temperature & 1.36 & 1.43 & 1.44 & 1.39 & 0.04 & 1.46 & 1.35 & 0.03 & 0.442 & 0.013 & 0.465 \\
\hline \multicolumn{12}{|l|}{ Color $^{8}$} \\
\hline $\mathrm{L}^{*}=$ lightness & $46.03^{b}$ & $47.49^{\mathrm{a}}$ & $45.05^{b}$ & $45.41^{b}$ & 0.45 & 46.20 & 45.80 & 0.33 & 0.030 & 0.499 & 0.603 \\
\hline$a^{*}=$ redness & $7.66^{\mathrm{a}}$ & $6.57^{b}$ & $7.09^{\mathrm{ab}}$ & $6.89^{b}$ & 0.21 & 7.00 & 7.13 & 0.16 & 0.030 & 0.595 & 0.392 \\
\hline$b^{*}=$ yellowness & -1.68 & -1.58 & -2.44 & -2.21 & 0.25 & -1.74 & -2.18 & 0.18 & 0.098 & 0.058 & 0.472 \\
\hline \multicolumn{12}{|l|}{ Loin quality traits 6} \\
\hline \multicolumn{12}{|l|}{ Color 8} \\
\hline $\mathrm{L}^{*}=$ lightness & 46.46 & 46.21 & 45.60 & 45.96 & 0.50 & 46.09 & 46.05 & 0.32 & 0.548 & 0.914 & 0.925 \\
\hline$a^{*}=$ redness & $6.12^{\mathrm{a}}$ & $5.46^{b}$ & $5.43^{b}$ & $5.60^{b}$ & 0.17 & 5.85 & 5.50 & 0.10 & 0.001 & 0.008 & 0.295 \\
\hline$b^{*}=$ yellowness & -1.84 & -2.03 & -2.29 & -1.98 & 0.26 & -1.78 & -2.25 & 0.18 & 0.610 & 0.028 & 0.948 \\
\hline Drip loss ( $\mathrm{g} \mathrm{kg}^{-1}$ ) & 43.0 & 42.2 & 36.1 & 38.9 & 0.48 & 38.9 & 41.6 & 0.26 & 0.574 & 0.423 & 0.819 \\
\hline Cooking loss ( $\mathrm{g} \mathrm{kg}^{-1}$ ) & 360.7 & 359.7 & 358.4 & 356.2 & 0.36 & 357.8 & 359.7 & 0.25 & 0.767 & 0.522 & 0.973 \\
\hline Shear force $(\mathrm{kg})^{8}$ & $4.63^{b}$ & $6.01^{\mathrm{a}}$ & $6.15^{\mathrm{a}}$ & $5.71^{\mathrm{a}}$ & 0.35 & 5.46 & 5.72 & 0.23 & 0.001 & 0.449 & 0.171 \\
\hline \multicolumn{12}{|l|}{ Marbling 6} \\
\hline Subjective score & 2.14 & 2.06 & 1.88 & 2.29 & 0.18 & 2.24 & 1.96 & 0.12 & 0.276 & 0.074 & 0.405 \\
\hline Intramuscular fat content $\left(\mathrm{g} \mathrm{kg}^{-1}\right)$ & 16.4 & 12.2 & 13.3 & 15.0 & 0.15 & 16.9 & 12.0 & 0.11 & 0.077 & $<0.001$ & 0.877 \\
\hline
\end{tabular}

${ }^{1} \mathrm{PC}=$ physically castrated (at age 7); IC = immunocastrated on $103^{\text {rd }}$ and $136^{\text {th }}$ day of life; ${ }^{2} \mathrm{RAC}=\mathrm{Ractopamine}-\mathrm{HCl}$ addition (at age 136 ); HCW = hot carcass weight; ; ${ }^{3}$ Considering $n=792$ pigs; ${ }^{4}$ Carcass yield $=(\mathrm{HCW} / \text { Final body weight })^{*} 1000 ;{ }^{5}$ Calculated from the HCW and backfat thickness, according to the equation: Percentage lean $=\mathrm{K}_{0}-\left(\mathrm{K}_{1}{ }^{*} \mathrm{BT}\right)+\left(\mathrm{K}_{2}{ }^{*} \mathrm{MD}\right)$ where $\mathrm{K}_{0}, \mathrm{~K}_{1}$ and $\mathrm{K}_{2}$ are constants from the commercial slaughterhouse where animals were slaughtered; ${ }^{6}$ Considering $\mathrm{n}=144$ pigs; ${ }^{7} \mathrm{pH}$ and temperature values were taken $45 \mathrm{~min}$ (initial) and $24 \mathrm{~h}$ after slaughter (ultimate); ${ }^{8}$ Color measurements were performed after a $24 \mathrm{~h}$ chilling period, instrumental color was determined on a center-cut SM slice after a 30 min bloom period. Shear force was determined after frozen LD chops were allowed to thaw for $24 \mathrm{~h}$ at $5{ }^{\circ} \mathrm{C}$ before samples were vacuum-packaged and cooked, chop thickness cubes were sheared perpendicular to the muscle fiber orientation; ${ }^{\text {ac Within }}$ a row, means without a common superscripts differ $(p<0.05)$ by Student's t-test (for ractopamine) and by F test (for RAC and castration main effects). 
Table 4 - Effect of castration categories ${ }^{1}$ and levels of RAC 2 in the diet on organs and pork cut yield.

\begin{tabular}{|c|c|c|c|c|c|c|c|c|c|c|c|}
\hline \multirow{2}{*}{ Item } & \multicolumn{4}{|c|}{ Ractopamine $\left(\mathrm{mg} \mathrm{kg}^{-1}\right)$} & \multirow{2}{*}{ SEM } & \multicolumn{2}{|c|}{ Castration category } & \multirow{2}{*}{ SEM } & \multicolumn{3}{|r|}{$p$-value } \\
\hline & 0 & 5 & 10 & 15 & & PC & IC & & RAC & Castration & RAC $\times$ Castration interaction \\
\hline \multicolumn{12}{|l|}{ Organs $^{3}$} \\
\hline Testis weight (g) & 693 & 697 & 656 & 658 & 33.65 & - & 676 & 17.10 & 0.769 & - & - \\
\hline Testis yield (g $100 \mathrm{~g}^{-1}$ ) & 0.57 & 0.57 & 0.54 & 0.54 & 0.25 & - & 0.55 & 0.01 & 0.678 & - & - \\
\hline Heart yield (g 100 g$^{-1}$ ) & 0.42 & 0.40 & 0.39 & 0.39 & 0.01 & 0.40 & 0.40 & 0.01 & 0.090 & 0.848 & 0.186 \\
\hline Liver yield (g $100 \mathrm{~g}^{-1}$ ) & $1.83^{\mathrm{ab}}$ & $1.88^{\mathrm{a}}$ & $1.69^{c}$ & $1.73^{\mathrm{bc}}$ & 0.04 & 1.68 & 1.88 & 0.03 & 0.010 & $<0.001$ & 0.330 \\
\hline Kidney yield (g $100 \mathrm{~g}^{-1}$ ) & 0.37 & 0.37 & 0.34 & 0.34 & 0.01 & 0.34 & 0.37 & 0.01 & 0.070 & 0.070 & 0.272 \\
\hline \multicolumn{12}{|l|}{ Cut yield (g $\left.100 \mathrm{~g}^{-1}\right)^{4}$} \\
\hline Jowl & 4.26 & 4.01 & 3.99 & 3.97 & 0.17 & 4.20 & 3.91 & 0.12 & 0.803 & 0.068 & 0.570 \\
\hline Ham & 25.41 & 26.03 & 26.10 & 25.56 & 0.39 & 25.56 & 26.00 & 0.27 & 0.468 & 0.566 & 0.342 \\
\hline Ham lean meat & 75.50 & 75.91 & 77.04 & 75.59 & 0.46 & 75.52 & 76.47 & 0.33 & 0.079 & 0.094 & 0.568 \\
\hline Ham fat & 4.79 & 4.24 & 3.96 & 4.48 & 0.40 & 4.91 & 3.83 & 0.26 & 0.356 & 0.017 & 0.319 \\
\hline Ham skin & 7.55 & 6.86 & 6.72 & 7.12 & 0.32 & 7.15 & 6.95 & 0.23 & 0.334 & 0.961 & 0.715 \\
\hline Ham bone & 12.16 & 13.00 & 12.28 & 12.81 & 0.38 & 12.41 & 12.75 & 0.27 & 0.456 & 0.673 & 0.396 \\
\hline Loin & 21.38 & 21.09 & 21.38 & 21.56 & 0.38 & 21.40 & 21.30 & 0.27 & 0.958 & 0.646 & 0.604 \\
\hline Loin lean meat & 52.97 & 55.34 & 55.39 & 55.32 & 1.07 & 54.58 & 55.05 & 0.81 & 0.376 & 0.920 & 0.292 \\
\hline Loin fat & 15.70 & 13.08 & 13.70 & 14.89 & 1.03 & 14.99 & 13.64 & 0.75 & 0.234 & 0.559 & 0.488 \\
\hline Loin skin & 4.35 & 4.56 & 4.37 & 3.67 & 0.26 & 4.18 & 4.27 & 0.20 & 0.438 & 0.926 & 0.632 \\
\hline Loin bone & 21.93 & 21.79 & 21.27 & 20.80 & 0.71 & 21.16 & 21.69 & 0.53 & 0.905 & 0.775 & 0.602 \\
\hline Belly + Ribs & 18.96 & 18.71 & 18.35 & 19.09 & 0.33 & 18.86 & 18.70 & 0.24 & 0.350 & 0.880 & 0.464 \\
\hline Belly lean meat & $63.26^{\mathrm{b}}$ & $64.53^{\mathrm{ab}}$ & $66.03^{a}$ & $65.44^{a}$ & 0.85 & 63.18 & 66.46 & 0.51 & 0.032 & $<0.001$ & 0.921 \\
\hline Belly fat & $13.47^{a}$ & $11.11^{\mathrm{b}}$ & $9.68^{b}$ & $11.81^{\mathrm{ab}}$ & 1.00 & 13.27 & 9.76 & 0.65 & 0.012 & $<0.001$ & 0.911 \\
\hline Belly skin & 8.85 & 9.66 & 8.95 & 8.55 & 0.33 & 9.12 & 8.91 & 0.50 & 0.234 & 0.726 & 0.880 \\
\hline Belly bone & 14.41 & 14.69 & 15.33 & 14.20 & 0.38 & 14.43 & 14.87 & 0.28 & 0.235 & 0.268 & 0.188 \\
\hline Shoulder & 18.60 & 19.15 & 18.80 & 18.50 & 0.02 & 18.81 & 18.73 & 0.17 & 0.209 & 0.508 & 0.074 \\
\hline Shoulder lean meat & 65.90 & 66.27 & 68.13 & 67.00 & 0.69 & 65.75 & 67.88 & 0.48 & 0.088 & 0.020 & 0.188 \\
\hline Shoulder fat & 10.96 & 10.18 & 9.19 & 10.60 & 0.73 & 11.46 & 9.04 & 0.47 & 0.404 & 0.010 & 0.775 \\
\hline Shoulder skin & 10.20 & 10.08 & 9.80 & 9.53 & 0.32 & 10.02 & 9.77 & 0.23 & 0.501 & 0.306 & 0.631 \\
\hline Boston butt & 8.41 & 8.42 & 8.77 & 8.38 & 0.25 & 8.49 & 8.50 & 0.18 & 0.626 & 0.685 & 0.355 \\
\hline Boston butt lean meat & 65.74 & 67.70 & 69.93 & 70.59 & 1.50 & 68.44 & 68.71 & 1.15 & 0.336 & 0.838 & 0.505 \\
\hline Boston butt fat & 5.05 & 2.90 & 3.17 & 3.71 & 0.44 & 3.88 & 3.48 & 0.38 & 0.056 & 0.549 & 0.823 \\
\hline
\end{tabular}

${ }^{\mathrm{I} P C}=$ physically castrated (at age 7); IC = immunocastrated on $103^{\text {rd }}$ and $136^{\text {th }}$ day of life; ${ }^{2} \mathrm{RAC}=$ Ractopamine-HCl addition (at age 136 ); ${ }^{3 R e l a t e d ~ t o ~ s l a u g h t e r ~}$ weight; Considering $n=792$ pigs; ${ }^{4}$ Related to hot carcass weight; Considering $n=72$ pigs.

yield: jowl, ham, ham lean meat, ham skin, ham bone, loin, loin lean meat, loin fat, loin skin, loin bone, belly and ribs, belly skin, belly bone, shoulder skin, Boston butt, Boston butt lean meat and Boston butt fat (Table 4). The RAC level also had no influence on ham fat yield, shoulder lean meat and shoulder fat yield ( $p>0.05)$. However, providing 10 or $15 \mathrm{mg} \mathrm{kg}^{-1}$ of RAC for pigs increased ( $p=$ 0.032 ) belly lean meat yield, whereas belly fat yield was reduced $(p=0.012)$ regardless of the RAC level. Ham fat yield $(p=0.017)$, belly fat yield $(p<0.001)$, and shoulder fat yield $(p<0.001)$ were all reduced in IC pigs, whereas belly lean meat yield $(p<0.001)$, and shoulder lean meat yield $(p=0.020)$ were increased.

\section{Discussion}

\section{Animal performance}

Growth requires a substantial quantity of nutrients to support tissue maintenance and deposition. Also, the growth rate of different body tissues depends on many factors such as animal age, genotype, sex, physiological maturity, body composition, initial and final body weight, level of feed intake, growth rate, thermal environment, immunological status and finally, feed processing and delivery (Patience et al., 2015). This may explain why no effect was observed among castration categories on performance characteristics in the initial phase (from 0 to 60 days).

In the growing-finishing period ( 61 to 136 days) less ADFI, higher ADG and better feed conversion was observed in the IC pigs, probably because effects of the first vaccine dose applied at d 103 had no physiological effect against GnRF on testis function. Therefore, the benefits may be related to the fact that immunocastrated pigs behave physiologically like intact boars until the second vaccine dose (Caldara et al., 2015). The natural anabolic potential of intact males positively affects the productive results in immunocastrated males, prior to the administration of the second vaccination, whereby these effects are lost. Rikard-Bell et al. (2009) commented that ADFI and ADG increased in the last two-week period after the second vaccination of IC pigs. This is why it is important 
to ensure the supplementation of RAC occurs either at the second vaccination stage or just prior, so that the full effect of RAC supplementation is able to offset the surge in ADFI and consequential increase in fat deposition often observed after the second vaccination.

The most predominant androgens in pig testis are $5 \alpha$-androstanediol, dehydro epiandrosterone (DHEA), 19-nortestosterone and testosterone. These steroids have anabolic effects that stimulate muscle and bone growth, nitrogen and phosphorus retention, and cause a redistribution of nutrients, resulting in better feed conversion, lower backfat thickness and increased lean tissue of intact male carcasses compared to castrates or females (Squires, 2011). In this study, in the time period from the second immunization dose at d 137 to $d 164$, there was no increase in ADFI of immunocastrated pigs, which was reported by Puls et al. (2014). However, other authors (Rikard-Bell et al., 2009; Fàbrega et al., 2010) have reported an increase in ADFI in immunocastrated males.

When immunocastrated pigs were fed RAC, they grew $18 \%$ faster than control intact males; also dietary RAC supplementation for the last $26 \mathrm{~d}$ before slaughter improved feed conversion ratio $(p=0.024)$ and ADG $(p$ $=0.046)$; also increased carcass total tissue $(p=0.023)$ and total lean $(p=0.027)$ content without affecting meat quality $(p>0.05)$ in both entire and immunocastrated male pigs (Moore et al., 2009).

Braña et al. (2013) observed no effect of gender $(p>0.05)$ on gain to feed ratio during nursery; however, surgical castrates grew faster $(p<0.05)$ than intact males and gilts and had greater $(p<0.05)$ ADFI than intact males. They reported no effect of castration category ( $p>0.05)$ on ADG in the growing period; however, IC had lower ADFI than PC and a greater gain to feed ratio than PC and gilts. During the finishing phase, both IC and PC had similar ADG and grew faster than gilts; feed conversion was better for IC than for PC. Supplementing finishing diets with 5 compared to $0 \mathrm{mg} \mathrm{kg}^{-1}$ RAC increased ADG and improved feed conversion but had no effect $(p>0.05)$ on carcass yield or fresh meat quality. Our results indicate that carcass yield was lower but percentage lean was greater for IC than for PC pigs.

The decrease in testosterone influences feeding activity with positive effects on growth performance (Dunshea et al., 2005). This fact corroborates increased feed intake that occurs after the application of the second vaccine dose, after which, nonetheless, ADFI was greater for castrated animals compared to immunocastrated males and females, but before slaughter the feed consumption of immunocastrated and castrated animals did not differ (Fàbrega et al., 2010).

Cai et al. (2015) observed that castration decreases serum testosterone levels and carcass leanness, resulting in a corresponding increase in carcass fat. Additionally, Cai et al. (2015) observed no difference in testosterone levels at 35 days of age. However, comparisons between castrated and intact males differed significantly at 84 ,
147 and $210 \mathrm{~d}$ of age. When the animal ages, testosterone levels rise. According to Carroll et al. (2005), castration is stressful independent of age; however, the stress associated with handling seems to increase with age.

Morales et al. (2013) when comparing immunocastrated males (IC), surgically castrated males (PC) and intact females (IF), observed that IC showed greater ADG for the entire period studied (d 87 to 164) and less ADFI, resulting in improved feed efficiency when compared to $\mathrm{PC}$ and IF.

Our results indicate that immunocastration had no major effect on ADFI, ADG and feed conversion from d 137 to 164 , because animals were submitted to feed restriction. This practice is very common in Brazil, but is not found in other countries, mainly due to labor. In our study, there was an increase in ADG in IC pigs from d 61 to 136, whereas ADFI decreased, resulting in an observed improvement in feed conversion and also a better slaughter weight in IC pigs compared to PC pigs. Throughout the finishing phase (d 137 to 164) all these parameters were not significantly different. However, RAC supplementation in pig diets during this period (the last $28 \mathrm{~d}$ of the finishing phase) promoted up to a $15 \%$ increase in ADG and improved feed conversion using 5, 10 or $15 \mathrm{mg} \mathrm{kg}^{-1}$ RAC.

Several authors such as Apple et al. (2007), Patience et al. (2009), Moore et al. (2009), and Rikard-Bell et al. (2009) have reported increased ADG and better feed conversion in finishing pigs fed RAC in their diet; these parameters were also improved in barrows and gilts according to Hinson et al. (2011) and Athayde et al. (2012).

A number of studies on boars, gilts and intact boars show decreases in fat deposition (Williams et al., 1994; Rikard-Bell et al., 2009), while others have shown no effect (Rincker et al., 2009; Ross et al., 2011) for this parameter.

It was demonstrated that RAC increases growth rapidly at the onset of feeding until a plateau is reached, after which there is a linear decline in growth response (Williams et al., 1994). It has been noted that the effect of RAC decreases as the duration of supply increases, as the $\beta$-adrenoceptors are downregulated within the fat tissue and/or desensitized in skeletal muscle tissue (Needham and Hoffman, 2015). Moore et al. (2009) evaluated feeding $5 \mathrm{mg} \mathrm{kg}{ }^{-1}$ RAC constantly for 26 days before slaughter vs. a step-up program where the same amount of RAC was fed for 14 days followed by 10 $\mathrm{mg} \mathrm{kg}{ }^{-1} \mathrm{RAC}$ for the final 12 days before slaughter in terms of growth performance, carcass composition and pork quality. Dietary RAC supplementation increased carcass total tissue and total lean content without affecting the objective meat quality in both intact and immunocastrated male pigs. However, a step-up program of RAC supplementation did not provide further improvements in growth performance and carcass composition when compared with constant $5 \mathrm{mg} \mathrm{kg}^{-1} \mathrm{RAC}$ supplementation. 


\section{Stress parameters}

An increase in blood lactate concentration was observed when $10 \mathrm{mg} \mathrm{kg}^{-1}$ of RAC were added to the diet, which suggests that these animals were more susceptible to stress that caused muscle hypertrophy. Under stress, there is intense muscle glycogen degradation, producing high levels of lactic acid that are released into the bloodstream. As RAC is similar to natural catecholamine, it may cause physiological changes in animals, inducing stress (Rocha et al., 2013). However, in this study, the cortisol levels in the blood were not altered by either immunocastration or the addition of RAC, suggesting that these treatments did not induce stress in the animals. These findings agree with Batorek et al. (2012).

\section{Carcass traits}

Castration causes pigs to become more docile; however, it also alters the metabolism leading to increased fat deposition in the carcass. Similarly, in this study we observed that the backfat thickness of PC males was greater than in the IC males, because the IC males were raised intact until vaccination, which allowed for an extension of the muscle deposition phase and a delay in carcass fat deposition, as reviewed by Dunshea et al. (2013).

Dietary RAC may decrease fat mass in intact and IC boars and offers an excellent means of maximizing the effects of immunocastration and minimizing the increase in fat mass sometimes observed in immunocastrated boars (Rikard-Bell et al., 2009). Although in our work, RAC inclusion did not affect pig backfat thickness. The absence of RAC effect may be related to restricted feeding during the finishing phase or genetically improved animals used in this study. These breeds are directed to meat production and low fat deposition, while feed restriction may provide lower energy availability to tissue synthesis. Additionally, Hinson et al. (2011) did not observe any RAC influence on fat thickness when evaluating barrows and gilts. According to Mills et al. (2003), RAC isomers have limited capacity to activate $\beta$-adrenergic receptors in adipose tissue of pigs, and the down-regulation may limit RAC effectiveness and lead to variation in carcass fat.

In our study, muscle depth was greater in pigs fed RAC, but was not influenced by castration categories. This result is in agreement with others (Fàbrega et al., 2010) who reported no immunocastration effect on muscle depth and Athayde et al. (2012) who reported increases in muscle depth of pigs fed RAC diets.

Beta-agonists are related to catecholamines which have a powerful pharmacological activity as tocolytic and bronchodilator agents. However, these compounds are also used as growth promoters in livestock production due to the anabolic effects exerted when higher doses are administered to animals (Blanca et al., 2005). It is not clearly elucidated how activation of $\beta$-adrenergic receptors increases protein deposition, but the evidence indicates these receptors mediate growth response (John- son et al., 2014). According to Sato et al. (2011), protein synthesis is, at least in part, mediated by $\beta 2$-adrenergic receptors in muscles and may explain the greater muscle depth of those animals fed RAC, as observed in the present study. With the exception of backfat thickness and muscle depth, all other evaluated carcass traits were influenced by both RAC levels and castration categories. The slaughter weight, HCW and percentage of lean meat were higher in immunocastrated males and also in pigs fed RAC in the diet. Rikard-Bell et al. (2009) observed that the half carcass of immunocastrated males was heavier than those from intact males or animals fed RAC. Athayde et al. (2012) also reported an increase in slaughter weight values, hot carcass and percentage of lean meat from pigs fed RAC in the diet. The favorable effect of immunocastration on lean meat percentage was also observed in several experiments: Moore et al. (2009), Fàbrega et al. (2010) and Braña et al. (2013).

\section{Quality parameters}

All values found for initial $\mathrm{pH}$ are within the standards (6.00-6.50) while ultimate $\mathrm{pH}$ values are lower (5.50-5.80) for pork; however, they were not different when compared with the addition of RAC or castration category. In a review made by Dunshea et al. (2005), it was reported that ultimate $\mathrm{pH}$ decreases in pigs supplemented with RAC (mainly by $20 \mathrm{mg} \mathrm{kg}^{-1}$ ) assuming that beta-agonists consume muscle glycogen, resulting in lactic acid accumulation in carcass after slaughter. However, Athayde et al. (2012) and Lowe et al. (2014) reported no effect on $\mathrm{pH}$ when 10 and $5 \mathrm{mg} \mathrm{kg}^{-1} \mathrm{RAC}$, respectively, were supplemented in the diet. Also Elsbernd et al. (2016) reported no effect on $\mathrm{pH}$ from different sex categories (immunocastrates, gilts, boars and physically castrates). According to Rincker et al. (2009), 5 mg kg-1 RAC minimally affected $\mathrm{pH}$ and objective color and has no effect on other pork quality measurements such as loin depth, fat depth or carcass lean percentage.

There was no effect of immunocastration on ham color parameters, although loin redness $\left(a^{*}\right)$ was lower for IC pigs. The addition of RAC influenced ham lightness $\left(\mathrm{L}^{*}\right)$, ham redness and loin redness, but showed no effect on drip loss, cooking loss, marbling and IMF content. Pigs fed RAC in their diet showed less red intensity in loin. The same occurred in IC pigs when compared to PC pigs. A decrease was observed in yellowness ( $\mathrm{b}^{*}$ ) associated with immunocastration, but no effect of RAC for this parameter. As mentioned by Garbossa et al. (2013), changes in b* value may be attributed to carotenoid pigments deposited in fat and also to fatty acid composition changes in IMF; in our study both loin yellowness and IMF decreased for IC pigs. As related by Apple et al. (2007), even if differences in pork color are reported, they are usually not visibly noticeable by consumers.

Redness is associated with higher amounts of iron in tissue and potassium; thus, higher $\mathrm{a}^{*}$ values mean higher quantities of myoglobin. In our study, somehow loin 
samples were less red for all RAC inclusion levels (from 5 to $15 \mathrm{mg} \mathrm{kg}^{-1}$ ), although in lightness all RAC levels were identical to the control samples, which is in agreement with most recent studies (Athayde et al., 2012; Garbossa et al., 2013; Lowe et al., 2014; Elsbernd et al., 2015).

The supplementation of RAC in diet increased loin shear force slightly above $5 \%$ regardless of RAC level, promoting less tenderness, which is in agreement with other authors that have studied RAC (Dunshea et al., 2005; Athayde et al., 2012), although Athayde et al. (2012) reported shear force increases when using $10 \mathrm{mg}$ $\mathrm{kg}^{-1}$ RAC. However, at $5 \mathrm{mg} \mathrm{kg}-1$ which is considered the lower level and most widely adopted level of RAC supplementation, no shear force influence was observed (Rincker et al., 2009; Athayde et al., 2012; Garbossa et al., 2013; Lowe et al., 2014).

When evaluating marbling, the subjective score showed no effect of addition of RAC. However, when pigs were immunized, they showed a tendency to have less marbling, probably because IC pigs spend most of their lives as intact males which deposit less fat (Asmus et al., 2014). IMF content was likely to decrease, especially when $5 \mathrm{mg} \mathrm{kg}^{-1}$ of RAC were added to the diet, probably due to rapid down regulation of the $\beta$-receptors in the fat tissue (Dunshea et al., 2005). IMF was also lower $(29 \%)$ in IC pigs, probably due to increases in diameter in muscle fibers associated with reduced lipogenesis and increased lipolysis of adipose tissue, which are specific actions of RAC (Williams et al., 1994).

\section{Organs and testis weight and yield}

IC pigs had their liver yield increased compared to PC pigs. Liver increase in IC pigs was also reported by a number of authors (Pauly et al., 2009; Asmus et al., 2014; Boler et al., 2014; Tavárez et al., 2014; Moore et al., 2016). As PC barrows have no testes, their steroid hormones are absent, allowing the liver to function more efficiently with regard to the metabolism of compounds such as skatole, thus affecting liver activity, which could influence liver weight. However, the effect of immunocastration on liver functioning is unclear (Needham and Hoffman, 2015).

Liver yield decreased when $10-15 \mathrm{mg} \mathrm{kg}^{-1}$ of RAC was used. It is possible that feed restriction reduced the weight of this organ and RAC itself at higher dosages may have influenced the liver metabolic activity, lacking energy for synthesis. As previously mentioned, RAC increases growth rapidly at the onset of feeding until a plateau is reached (usually in the first seven days), after which there is a gradual decline in growth response, from the second to the fourth week after the administration of RAC (Williams et al., 1994). No further evidence was reported in the literature regarding liver decrease when pigs were fed with RAC. However, similar to the observations of this study, RAC supplementation has been reported to increase liver yield (Paulk et al., 2015). More studies should be conducted to elucidate this fact.

\section{Carcass cutting yield}

In our study, only belly lean meat and belly fat were influenced by RAC. As regards immunocastration, other variables including those affected by RAC also diverged $(p<0.05)$ such as ham fat, shoulder lean meat and shoulder fat (Table 4). When $15 \mathrm{mg} \mathrm{kg}^{-1} \mathrm{RAC}$ were added to diet, an increase of slightly above $2 \%$ in belly lean meat was observed. Belly fat decreased by almost $2 \%$ when compared to the control group, although there was no difference $(p<0.001)$ in the amount of RAC 15 , 10 or $15 \mathrm{mg} \mathrm{kg}^{-1}$ ) on these variables.

Currently, only limited data is available on carcass cutting yield of IC fed RAC as pointed out by Needham and Hoffman (2015). When the effect of RAC on the cutting yield of IC is evaluated, feeding RAC has little effect on the whole shoulder, loin, ham, and belly when expressed as a percentage of the HCW (Lowe et al., 2014). The only whole cut percentage influenced by immunocastration according to Needham and Hoffman (2015) was the belly while Rikard-Bell et al. (2009) observed differences $(p<0.05)$ in lean and fat deposition in shoulder, loin, and belly when RAC was added to the diet. Furthermore, these authors considered the effect of sex (gilts, boars and immunocastrates) and observed that cuts as shoulder and ham were both significant $p<$ 0.05 ) in lean and fat deposition.

Differences between IC and PC pigs were observed for carcass cuts (Table 4). Ham yield were reduced ( $p=0.017$ ) by nearly $1 \%$ and belly lean meat increased $(p<0.001)$ with a corresponding decrease in belly fat $(p$ $<0.001)$, shoulder lean meat increased by $2 \%(p=0.02)$ and shoulder fat was reduced $(p=0.01)$ by approximately $2 \%$ for IC pigs when compared to PC pigs. Tavárez et al. (2014) have found less lipid content (75 vs. 83 \%) for IC than PC barrows in belly adipose tissue and observed that after the second vaccination dose, lipid jowl increased, but lipid belly remained unchanged.

Both RAC (at least $5 \mathrm{mg} \mathrm{kg}^{-1}$ supplementation) and immunological castration were additive in terms of improving carcass cutting yield while having minimal effects on pork quality. These findings are in agreement with Lowe et al. (2014) and Rikard-Bell et al. (2009).

\section{Acknowledegments}

The authors wish to acknowledge Dr. Antonio L. Guidoni (in memoriam) for the statistical analysis of the data, Cooperativa Central Aurora Alimentos (Chapecó, SC) and Coperdia (Concordia, SC) for partnership and also Zoetis/Pfizer for the GnRF vaccine donation.

\section{References}

\footnotetext{
Aaslyng, M.D.; Broge, E.H.D.L.; Brockhoff, P.B.; Christensen, R.H.B. 2016. The effect of skatole and androstenone on consumer response towards fresh pork from $\mathrm{m}$. longissimusthoracis et lumborum and $\mathrm{m}$. semimembranosus. Meat Science 116: 174-185.
} 
American Meat Science Association [AMSA]. 1995. Research Guidelines for Cookery, Sensory and Instrumental Tenderness Measurement of Fresh Meat. American Meat Science Association, Chicago, IL, USA.

Apple, J.K.; Rincker, P.J.; McKeith, F.K.; Carr, S.N.; Armstrong, T.A.; Matzat, P.D. 2007. Meta-analysis of the ractopamine response in finishing swine. Professional Animal Scientist 23: 179-196.

Asmus, M.D.; Tavarez, M.A.; Tokach, M.D.; Dritz, S.S.; Schroeder, A.L.; Nelssen, J.L.; Goodband, R.D.; DeRouchey, J.M. 2014. The effects of immunological castration and corn dried distillers grains with solubles withdrawal on growth performance, carcass characteristics, fatty acid analysis, and iodine value of pork fat depots. Journal of Animal Science 92: 2116-2132.

Association of Official Analyst Chemists [AOAC]. 2007. Official Methods of Analysis of AOAC International. AOAC International, Gaithersburg, MD, USA.

Athayde, N.B.; Dalla Costa, O.A.; Roca, R.O.; Guidoni, A.L.; Ludtke, C.B.; Lima, G.J.M.M. 2012. Meat quality of swine supplemented with ractopamine under commercial conditions in Brazil. Journal of Animal Science 90: 4604-4610.

Batorek, N.; Škrlep, M.; Prunier, A.; Louveau, I.; Noblet, J.; Bonneau, M.; andek-Potokar, M. 2012. Effect of feed restriction on hormones, performance, carcass traits, and meat quality in immunocastrated pigs. Journal of Animal Science 90: 4593-4603.

Boler, D.D.; Puls, C.L.; Clark, D.L.; Ellis, M.; Schroeder, A.L.; Matzat, P.D.; Killefer, J.; McKeith, K.; Dilger, A.C. 2014. Effects of immunological castration (Improvest) on changes in dressing percentage and carcass characteristics of finishing pigs. Journal of Animal Science 91: 359-368.

Braña, D.V.; Rojo-Gómez, G.A.; Ellis, M.; Cuaron, J.A. 2013. Effect of gender (gilt and surgically and immunocastrated male) and ractopamine hydrochloride supplementation on growth performance, carcass, and pork quality characteristics of finishing pigs under commercial conditions. Journal of Animal Science 91: 5894-904.

Cai, Z.; Zhang, L.; Jiang, X.; Sheng, Y.; Xu, N. 2015. Differential miRNA expression profiles in the longissimus dorsi muscle between intact and castrated male pigs. Research in Veterinary Science 99: 99-104.

Caldara, F.R.; Santos, R.K.; Santos, L.S.; Foppa, L.; Nääs, I.A.; Garcia, R.G.; Machado, S.P. 2015. Performance and plasma urea nitrogen of immunocastrated males pigs of medium genetic potential. Revista MVZ Córdoba 20: 4572-4580.

Carroll, J.A.; Berg, E.L.; Strauch, T.A.; Roberts, M.P.; Kattesh, H.G. 2005. Hormonal profiles, behavioral responses, and shortterm growth performance after castration of pigs at three, six, nine, or twelve days of age. Journal of Animal Science 84: 1271-1278.

Cromwell, G.L. 2009. ASAS centennial paper: landmark discoveries in swine nutrition in the past century. Journal of Animal Science 87: 778-792.

Dunshea, F.R.; Allison, J.R.D.; Bertram, M.; Boler, D.D.; Brossard, L.; Campbell, R.; Crane, J.P.; Hennessy, D.P.; Huber, L.; De Lange, C.; Ferguson, N.; Matzat, P.; McKeith, F.; Moraes, P.J.U.; Mullan, B.P.; Noblet, J.; Quiniou, N.; Tokach, M. 2013. The effect of immunization against $\mathrm{GnRF}$ on nutrient requirements of male pigs: a review. Animal 7: 1769-1778.
Dunshea, F.R.; D'Souza, D.N.; Pethick, D.W.; Harper, G.S.; Warner, R.D. 2005. Effects of dietary factors and other metabolic modifiers on quality and nutritional value of meat. Meat Science 71: 8-38.

Elsbernd, A.J.; Patience, J.F.; Prusa, K.J. 2016. A comparison of the quality of fresh and frozen pork from immunologically castrated males versus gilts, physical castrates, and entire males. Meat Science 111: 110-115.

Elsbernd, A.J.; Stalder, K.J.; Karriker, L.A.; Patience, J.F. 2015. Comparison among gilts, physical castrates, entire males, and immunological castrates in terms of growth performance, nitrogen and phosphorus retention, and carcass fat iodine value. Journal of Animal Science 93: 5702-5710.

Fàbrega, E.; Velarde, A.; Cros, J.; Gispert, M.; Suárez, P.; Tibau, J.; Soler, J. 2010. Effect of vaccination against gonadotrophinreleasing hormone, using Improvac ${ }^{\circledR}$, on growth performance, body composition, behaviour and acute phase proteins. Livestock Science 132: 53-59.

Garbossa, C.A.P.; Sousa, R.V.; Cantarelli, V.S.; Pimenta, M.E.S.; Zangeronimo, M.G.; Silveira, H.; Kuribayashi, T.H.; Cerqueira, L.G.S. 2013. Ractopamine levels on performance, carcass characteristics and quality of pig meat. Revista Brasileira de Zootecnia 42: 325-333.

Hinson, R.B.; Wiegand, B.R.; Ritter, M.J.; Allee, G.L.S.; Carr, N. 2011. Impact of dietary energy level and ractopamine on growth performance, carcass characteristics, and meat quality of finishing pigs. Journal of Animal Science 89: 3572-3579.

Honikel, K.O. 1998. Reference methods for the assessment of physical characteristics of meat. Meat Science 49: 447-457.

Johnson, B.J.; Smith, S.B.; Chung, K.Y. 2014. Historical overview of the effect of $\beta$-adrenergic agonists on beef cattle production. Asian-Australasian Journal of Animal Sciences 27: 757-766.

Lowe, B.K.; Gerlemann, G.D.; Carr, S.N.; Rincker, P.J.; Schroeder, A.L.; Petry, D.B.; McKeith, F.K.; Allee, G.L.; Dilger, A.C. 2014. Effects of feeding ractopamine hydrochloride (Paylean) to physical and immunological castrates (Improvest) in a commercial setting on carcass cutting yields and loin quality. Journal of Animal Science 92: 3715-3726.

Meier-Dinkel, L.; Trautmann, J.; Frieden, L.; Tholen, E.; Knorr, C.; Sharifi, A.R.; Bücking, M.; Wicke, M.; Mörlein, D. 2013. Consumer perception of boar meat as affected by labelling information, malodorous compounds and sensitivity to androstenone. Meat Science 93: 248-256.

Mills, S.E.; Spurlock, M.E.; Smith, D.J. 2003. B-adrenergic receptor. Journal of Animal Science 81: 662-668.

Moore, K.L.; Dunshea, F.R.; Mullan, B.P.; Hennessy, D.P.; D'Souza, D.N. 2009. Ractopamine supplementation increases lean deposition in entire and immunocastrated male pigs. Animal Production Science 49: 1113-1119.

Moore, K.L.; Mullan, B.P.; Kim, J.C.; Dunshea, F.R. 2016. Standardized ileal digestible lysine requirements of male pigs immunized against gonadotrophin releasing factor. Journal of Animal Science 94: 1982-1992.

Morales, J.I.; Serrano, M.P.; Cámara, L.; Berrocoso, J.D.; López, J.P.; Mateos, G.G. 2013. Growth performance and carcass quality of immunocastrated and surgically castrated pigs from crossbreds from Duroc and Pietrain sires. Journal of Animal Science 91: 3955-3964. 
Needham, T.; Hoffman, L.C. 2015. Carcass traits and cutting yields of entire and immunocastrated pigs fed increasing protein levels with and without ractopamine hydrochloride supplementation. Journal of Animal Science 93: 4545-4556.

National Pork Producers Council [NPPC]. 1999. Pork Quality Standards. NPPC, Des Moines, IA, USA.

Patience, J.F.; Rossoni-Serão, M.C.; Gutiérrez, N.A. 2015. A review of feed efficiency in swine: biology and application. Journal of Animal Science \& Biotechnology 6: 1-9.

Patience, J.F.; Shand, P.; Pietrasik, Z.; Merrill, J.; Vessie, G.; Ross, K.A.; Beaulieu, A.D. 2009. The effect of ractopamine supplementation at $5 \mathrm{ppm}$ of swine finishing diets on growth performance, carcass composition and ultimate pork quality. Canadian Journal of Animal Science 89: 53-66.

Paulk, C.B.; Burnett, D.D.; Tokach, M.D.; Nelssen, J.L.; Dritz, S.S.; DeRouchey, J.M.; Goodband, R.D.; Hill, G.M.; Haydon, K.D.; Gonzalez, J.M. 2015. Effect of added zinc in diets with ractopamine hydrochloride on growth performance, carcass characteristics, and ileal mucosal inflammation mRNA expression of finishing pigs. Journal of Animal Science 93: 185196.

Pauly, C.; Spring, P.; O'Doherty, J.V.; Ampuero Kragten, S.; Bee, G. 2009. Growth performance, carcass characteristics and meat quality of group-penned surgically castrated, immunocastrated $\left(\right.$ Improvac $\left.^{\circledR}\right)$ and entire male pigs and individually penned entire male pigs. Animal 3: 1057-1066.

Puls, C.L.; Rojo, A.; Ellis, M.; Boler, D.D.; McKeith, F.K.; Killefer, J.; Gaines, A.M.; Matzat, P.D.; Schroeder, A.L. 2014. Growth performance of immunologically castrated (with Improvest) barrows (with or without ractopamine) compared to gilt, physically castrated barrow, and intact male pigs. Journal of Animal Science 92: 2289-2295.

Rikard-Bell, C.; Curtis, M.A.; van Barneveld, R.J.; Mullan, B.P.; Edwards, A.C.; Gannon, N.J.; Henman, D.J.; Hughes, P.E.; Dunshea, F.R. 2009. Ractopamine hydrochloride improves growth performance and carcass composition in immunocastrated boars, intact boars, and gilts. Journal of Animal Science 87: 3536-3543.
Rincker, P.J.; Killefer, J.; Matzat, P.D.; Carr, S.N.; McKeith, F.K. 2009. The effect of ractopamine and intramuscular fat content on sensory attributes of pork from pigs of similar genetics. Journal of Muscle Foods 20: 79-88.

Rocha, L.M.; Bridi, A.M.; Foury, A.; Mormede, P.; Weschenfelder, A.V.; Devillers, N.; Bertoloni, W.; Faucitano, L. 2013. Effects of ractopamine administration and castration method on the response to preslaughter stress and carcass and meat quality in pigs of two Pietrain genotypes. Journal of Animal Science 91: 3965-3977.

Ross, K.A.; Beaulieu, A.D.; Merrill, J.; Vessie, G.; Patience, J.F. 2011. The impact of ractopamine hydrochloride on growth and metabolism, with special consideration of its role on nitrogen balance and water utilization in pork production. Journal of Animal Science 89: 2243-2256.

Rostagno, H.S.; Albino, L.F.T.; Donzele, J.L.; Gomes, P.C.; Oliveira, R.F.; Lopes, D.C.; Ferreira, A.S.; Barreto, S.L.T. 2011. Brazilian Tables for Poultry and Swine: Composition of Feedstuffs and Nutritional Requirements. 3ed. Federal University of Viçosa, Viçosa, MG, Brazil.

Sato, S.; Shirato, K.; Tachiyashiki, K.; Imaizumi, K. 2011. Muscle plasticity and $\beta 2$-adrenergic receptors: adaptive responses of $\beta$. Journal of Biomedicine and Biotechnology 2011: 1-10.

Squires, E.J. 2011. Applied Animal Endocrinology. CABI, Wallingford, UK.

Tavárez, M.A.; Puls, C.L.; Schroeder, A.L.; Dilger, A.C. 2014. Effects of immunocastration and time after second Improvest dose on adipose tissue fatty acid profile of finishing barrows. Journal of Animal Science 92: 3736-3744.

Ulrey, W.D.; Burnett, T.J.; Brunelle, S.L.; Lombardi, K.R.; Coleman, M.R. 2013. Determination and confirmation of parent and total ractopamine in bovine, swine, and turkey tissues by liquid chromatography with tandem mass spectrometry: final action 2011.23. Journal of AOAC International 96: 917-924.

Williams N.H.; Cline, T.; Schinckel, A. 1994. The impact of ractopamine, energy intake, and dietary fat on finisher pig growth performance and carcass merit. Journal of Animal Science 72: 3152-3162. 